\title{
Modeling and Simulation of Laparoscopic Tools for Autonomously Positioning Laparoscope in Laparoscopic Surgery
}

\author{
S. M. Megahed, A. A. Balbola \\ Mechanical Design \& Production Engineering Department, Faculty of Engineering, Giza, Egypt \\ Email: smegahed@cu.edu.eg, amrbalbola@cu.edu.eg
}

Received December 2012

\begin{abstract}
In laparoscopic surgery, the surgeons are equipped with the suitable tools for the surgery, while the laparoscope is used to capture the operation environment and displays it on a monitor. This paper presents the mathematical kinematic position modeling of the laparoscopic tools used for autonomous positioning of a laparoscope in such operations. These models are obtained using Denavit-Hartenberg (D-H) Notations and Homogenous Transformation Matrix (HTM). The laparoscopic tools are considered as six degrees of freedom (DOF) mechanisms while the laparoscope has four DOF. The 3D loop closure equation is used to obtain the laparoscope kinematic position models in terms of those of the laparoscopic tools. These models are used to simulate and align the laparoscope camera with the surgeon's laparoscopic Tools Center Points (TCP). The obtained results show the smooth positioning of the laparoscope camera for better visualization of laparoscopic surgery environments.
\end{abstract}

Keywords: Laparoscopic Surgery; Homogenous Transformation Matrix; Denavit-Hartenberg Notations

\section{Introduction}

In laparoscopic surgery, two main problems challenge the surgeons. The first is increasing field of vision capacity and controlling system to carry out complex operational gestures without using high body mobility. The second is the tools flexibilities inside the human body to do more complex in vivo healthcare operations. Now most Laparoscopic Surgeries are done using 6 DOF laparoscopic tools to increase their flexibility inside the human body. Surgeons visualize the surgery environment using a laparoscope. Any wrong movement of the laparoscope interrupts the operation and the surgeon stops until controlling the laparoscope focus again on the operation area. Many researches are done for autonomously positioning the laparoscope instead of the laparoscope manual positioning by an operator. Halin et al. [1] developed a novel a laparoscope which detect and to follow the movements of the surgeon's head. Terry et al. [2] built a port camera integrated with the laparoscopic tool. Weede et al. [3] designed an endoscopic guidance system to autonomously align the laparoscope with the TCP of the surgeon's instruments which uses the information on the movements of the instruments from former interventions to predict the laparoscope motion. Terry et al. also [4] designed, built, and tested a novel single-port access laparoscopic surgery specific camera system (magnet camera). Xu et al. [5] derives the analytical solution for a 5-DOF manipulator to follow a given trajectory.

In this paper, the direct and inverse kinematic position models of two laparoscopic tools and one laparoscope are derived for autonomous positioning of the laparoscope camera. After an introduction, the paper is organized in three main sections. In Section 1, the kinematic position models of the two 6 DOF laparoscopic tools and one laparoscope are presented. In Section 2, the laparoscope models are developed as function of the parameters of the two laparoscopic tools. The obtained simulation results using the developed models are presented in Section 3.

\section{Kinematic Modeling of 6 DOF Laparoscopic Tools}

In laparoscopic surgery, Surgeons use two 6 DOF laparoscopic tools (right $\&$ left) to carry out complex operations. The four Denavit-Hartenberg (D-H) parameters $\left(\theta_{i}\right.$, $r_{i}, a_{i}$, and $\alpha_{i}$ for $i=1,2 \cdots 6$ ) are used for generating the elementary HTM's $\left(T_{i, i+1}\right)$ of the laparoscopic tools (see Figure 1, and Table 1) [6].

By substituting the D-H parameters in Table 1 into (1) the 6 elementary HTM's $\left(T_{i, i+1}\right)$ for $i=1,2 \cdots 6$ are determined which are used in (2) to obtain the generalized $\operatorname{HTM}\left(T_{0,7}\right)$. 


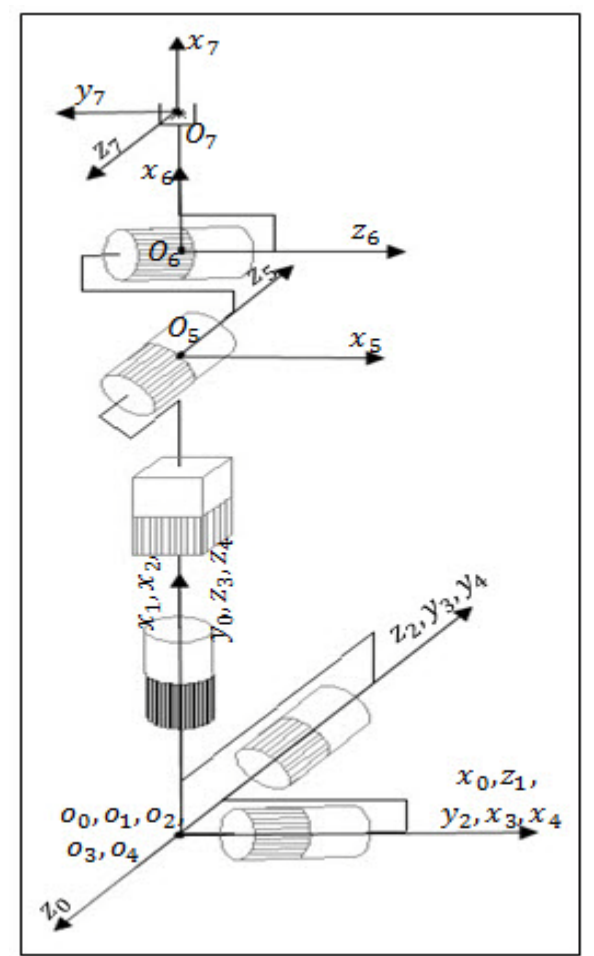

Figure 1. Laparoscopic tool kinematic diagram.

Table 1. D-H parameters.

\begin{tabular}{cccccccc}
\hline $\begin{array}{c}\text { Joint i } \\
\text { Parameter s }\end{array}$ & $\mathbf{0}$ & $\mathbf{1}$ & $\mathbf{2}$ & $\mathbf{3}$ & $\mathbf{4}$ & $\mathbf{5}$ & $\mathbf{6}$ \\
\hline $\boldsymbol{\sigma}_{i}$ & - & 0 & 0 & 0 & 1 & 0 & 0 \\
$\theta_{i}$ & $\frac{\pi}{2}$ & $q_{1}$ & $q_{2}$ & $q_{3}$ & 0 & $q_{3}$ & $q_{6}$ \\
$r_{i}$ & 0 & 0 & 0 & 0 & $q_{4}$ & 0 & 0 \\
$a_{i}$ & 0 & 0 & 0 & 0 & 0 & $\mathrm{a}_{5}$ & $\mathrm{a}_{6}$ \\
$\alpha_{i}$ & $\frac{\pi}{2}$ & $\frac{\pi}{2}$ & $\frac{\pi}{2}$ & 0 & $-\frac{\pi}{2}$ & $-\frac{\pi}{2}$ & $-\frac{\pi}{2}$ \\
\hline
\end{tabular}

This generalized HTM is used with (3) representing the desired laparoscopic tool generalized HTM $\left(T_{0,7}\right)$ to obtain the expressions of the laparoscopic tool direct kinematic position model (DKPM) and the position and orientation of its TCP are obtained. The inverse kinematic position model (IKPM) is also derived using the same generalized HTM. In the next equations $S=\sin$ and $C=$ cos.

Elementary HTM:

$$
T_{i, i+1}=\left[\begin{array}{cccc}
C C \theta_{i} & -S S \theta_{i} C C \alpha_{i} & S S \theta_{i} S S \alpha_{i} & a_{i} C C \theta_{i} \\
S S \theta_{i} & C C \theta_{i} C C \alpha_{i} & -C-C \theta_{i} S S \alpha_{i} & a_{i} S S \theta_{i} \\
0 & S S \alpha_{i} & C C \alpha_{i} & r_{i} \\
0 & 0 & 0 & 1
\end{array}\right]
$$

Generalized HTM:

$$
\begin{gathered}
T_{0,7}=\prod_{i=1}^{7} \mathrm{~T}_{i-1, i} \\
T_{0,7}=\left[\begin{array}{cccc}
r_{11} & r_{12} & r_{13} & n \\
r_{21} & r_{22} & r_{23} & m \\
r_{31} & r_{32} & r_{33} & l \\
0 & 0 & 0 & 1
\end{array}\right]
\end{gathered}
$$

DKPM \& IKPM: An algebraic approach is used to calculate the laparoscopic tool DKPM and IKPM as follows:

First (2) is pre-multiplied by the inverse of the HTM $\left(T_{0,3}\right)$ as presented in (4) resulting 6 Equations $(5,6,7,8$, 9 , and 10). Second (2) is pre-multiplied by the inverse of the HTM $\left(T_{0,2}\right)$ and post-multiplied by the inverse of the $\operatorname{HTM}\left(T_{6,7}\right)$ as presented in (11) resulting 2 Equations (12, 13). The obtained eight equations are used to calculate the expressions of the laparoscopic tool DKPM which are solved to calculate its IKPM.

$$
\begin{aligned}
& \left(T_{0,1} \cdot T_{1,2} \cdot T_{2,3}\right)^{-1} \cdot T_{0,7}=T_{3,4} \cdot T_{4,5} \cdot T_{5,6} \cdot T_{6,7} \\
& r_{21} \cdot S q_{1}-r_{31} \cdot C q_{1}=S q_{3} \cdot C q_{5} \cdot C q_{6}-C q_{3} \cdot S q_{6} \\
& r_{22} \cdot S q_{1}-r_{32} \cdot C q_{1}=S q_{3} \cdot C q_{5} \\
& m \cdot S q_{1}-n \cdot C q_{1} \\
& =a_{6} \cdot\left(S q_{3} \cdot C q_{5} \cdot C q_{6}-C q_{3} \cdot S q_{6}\right)+a_{5} \cdot S q_{3} \cdot C q_{5} \\
& r_{11} \cdot C q_{2}-S q_{2} \cdot\left(r_{21} \cdot C q_{1}+r_{31} \cdot S q_{1}\right)=S q_{5} \cdot C q_{6} \\
& -r_{13} \cdot C q_{2}+S q_{2} \cdot\left(r_{23} \cdot C q_{1}+r_{33} \cdot S q_{1}\right)=S q_{5} \cdot S q_{6} \\
& l \cdot C q_{2}-S q_{2} \cdot\left(m \cdot C q_{1}+n \cdot S q_{1}\right) \\
& =a_{6} \cdot S q_{5} \cdot C q_{6}+a_{5} \cdot S q_{5}-q_{4} \\
& \left(T_{0,1} \cdot T_{1,2}\right)^{-1} \cdot T_{0,7} \cdot T_{6,7}^{-1}=T_{2,3} \cdot T_{3,4} \cdot T_{4,5} \cdot T_{5,6} \\
& C q_{6} \cdot\left(r_{21} \cdot S q_{1}-r_{31} \cdot S q_{1}\right) \\
& +S q_{6} \cdot\left(r_{23} \cdot S q_{1}-r_{33} \cdot C q_{1}\right)=S q_{3} \cdot C q_{5} \\
& S q_{6} \cdot\left(r_{21} \cdot S q_{1}-r_{31} \cdot S q_{1}\right) \\
& +C q_{6} \cdot\left(r_{23} \cdot S q_{1}-r_{33} \cdot C q_{1}\right)=-S q_{3} \cdot S q_{5}
\end{aligned}
$$

The following is the IKPM of the 6 DOF laparoscopic tools

$$
\begin{aligned}
& a_{1}=\frac{m^{2}}{a_{5}^{2}}+\left(\frac{a_{6}^{2}}{a_{5}^{2}}-1\right) \cdot r_{21}^{2}-\frac{2 a_{6} \cdot m}{a_{5}^{2}} \cdot r_{21}+r_{22}^{2}-r_{23}^{2} \\
& b_{1}=-\frac{2 m}{a_{5}^{2}}-2\left(\frac{a_{6}^{2}}{a_{5}^{2}}-1\right) \cdot r_{21} \cdot r_{31}+\frac{2 a_{6}}{a_{5}^{2}}\left(m \cdot r_{31}+n \cdot r_{21}\right) \\
& -2 r_{22} \cdot r_{32}+2 r_{23} \cdot r_{33}
\end{aligned}
$$




$$
\begin{aligned}
& c_{1}=\frac{n^{2}}{a_{5}^{2}}+\left(\frac{a_{6}^{2}}{a_{5}^{2}}-1\right) r_{31}^{2}-\frac{2 a_{6} \cdot n}{a_{5}^{2}} r_{31}+r_{32}^{2}-r_{33}^{2} \\
& q_{1}=\operatorname{atanatan}\left(\frac{-b_{1} \pm \sqrt{b_{1}^{2}-4 a_{1} c_{1}}}{2 a_{1}}\right) \\
& d_{61}=r_{23} \cdot S q_{1}-r_{32} \cdot C q_{1} \\
& d_{62}=r_{21} \cdot S q_{1}-r_{31} \cdot C q_{1} \\
& c_{61}=r_{32} \cdot C q_{1}-r_{22} \cdot S q_{1} \\
& c_{62}=\frac{m \cdot S_{1} \text { qn } \cdot C q_{1}-a_{6} \cdot d_{12}}{a_{5}} \\
& {\left[\begin{array}{l}
C q_{6} \\
S q_{6}
\end{array}\right]=\left[\begin{array}{cc}
d_{61} & d_{62} \\
d_{62} & -d_{61}
\end{array}\right]^{-1}\left[\begin{array}{c}
c_{61} \\
c_{62}
\end{array}\right]} \\
& q_{6}=\operatorname{atan} 2\left(S q_{6}, C q_{6}\right) \\
& q_{5}=\operatorname{atan}\left(\frac{-d_{61}}{d_{62}}\right) \\
& {\left[\begin{array}{l}
S q_{3} \\
C q_{3}
\end{array}\right]=\left[\begin{array}{cc}
C q_{5} & 0 \\
C q_{5} * C q_{6} & -S q_{6}
\end{array}\right]^{-1}\left[\begin{array}{l}
c_{62} \\
d_{62}
\end{array}\right]} \\
& q_{3}=\operatorname{atan} 2\left(S q_{3}, C q_{3}\right) \\
& d_{41}=m \cdot C q_{1}+n \cdot S q_{1} \\
& q_{4}=-l \cdot C q_{2}+S q_{2} \cdot d_{41}+c_{41} \\
& c_{41}=a_{6} \cdot S q_{5} \cdot C q_{6}+a_{5} \cdot S q_{5} \\
& c_{42}=a_{6} \cdot\left(C q_{3} \cdot C q_{5} \cdot C q_{6}+S q_{3} \cdot S q_{6}\right) \\
& +a_{5} \cdot C q_{3} \cdot C q_{5} \\
& {\left[\begin{array}{l}
S q_{2} \\
C q_{2}
\end{array}\right]=\left[\begin{array}{cc}
d_{41} & -l \\
l & d_{41}
\end{array}\right]^{-1}\left[\begin{array}{c}
q_{4}-c_{41} \\
c_{42}
\end{array}\right]} \\
& q_{3}=\operatorname{atan} 2\left(S q_{3}, C q_{3}\right)
\end{aligned}
$$

\section{Positioning of a Robot Assisted Surgery Laparoscope}

It's supposed that the laparoscope is always tracking the midpoint between the TCPs of the right and left laparoscopic tools. The 3D loop closure theory is used to get the HTM of the laparoscope in terms of the left and right laparoscopic tools joint angles [7]. Figures 2 and 3 present the closed loops formed by the HTM of the right, left laparoscopic tool, and laparoscope. The product of these HTMs is equal to a unity matrix as in (33) and (39).

$$
T_{A D}=T_{A B} T_{B C} T_{C D}
$$

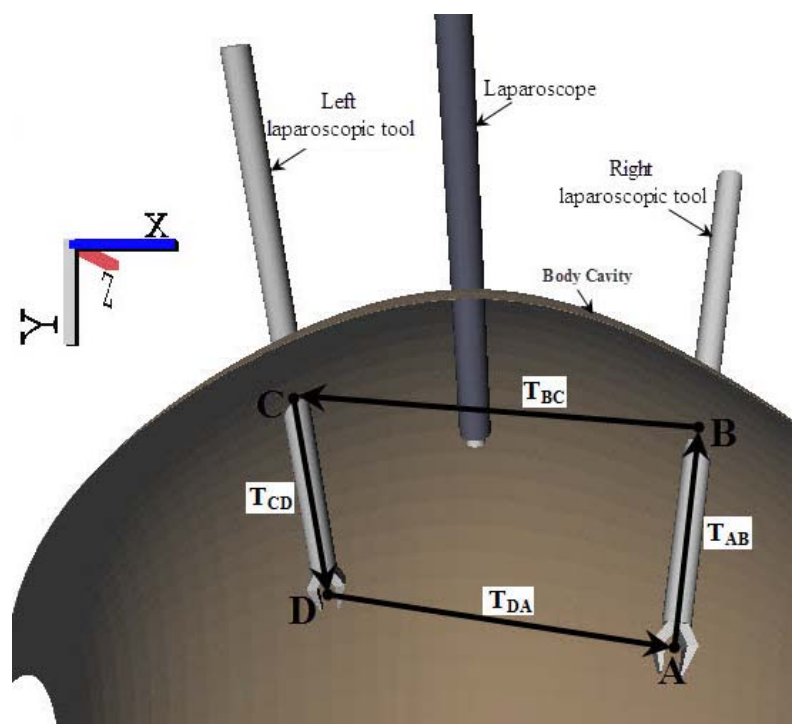

Figure 2. Laparoscopic surgery environment.

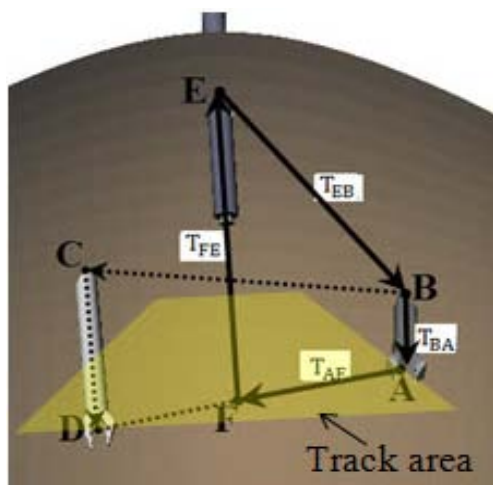

Figure 3. Surgery area.

$$
\begin{aligned}
T_{B A} & =\left[\begin{array}{cc}
R_{b a(3 \times 3)} & P_{b a(3 \times 1)} \\
0_{(1 \times 3)} & 1
\end{array}\right] \\
T_{A B} & =\left[\begin{array}{cc}
R_{b a(3 \times 3)}^{t} & -R_{b a(3,3)}^{t} P_{b a(3 \times 1)} \\
0_{(1 \times 3)} & 1
\end{array}\right] \\
T_{C D} & =\left[\begin{array}{cc}
R_{c d(3 \times 3)} & P_{c d(3 \times 1)} \\
0_{(1 \times 3)} & 1
\end{array}\right] \\
T_{B C} & =\left[\begin{array}{cc}
\mathrm{IP} & \mathrm{bc} \\
0_{(1 \times 3)} & 1
\end{array}\right] \\
T_{A D} & =\left[\begin{array}{cc}
R_{b a}^{t} R_{c d} & R_{b a}^{t}\left(P_{b c}+P_{c d}\right)-R_{b a}^{t} P_{b a} \\
0_{(1 \times 3)} & 1
\end{array}\right] \\
T_{E F} & =T_{E B} T_{B A} T_{A F} \\
T_{E B} & =\left[\begin{array}{cc}
\mathrm{I} & P_{e b(3 \times 1)} \\
0_{(1 \times 3)} & 1
\end{array}\right]
\end{aligned}
$$




$$
\begin{gathered}
T_{A F}=\left[\begin{array}{cc}
R_{b a}^{t} R_{c d} & r \cdot\left[R_{b a}^{t}\left(P_{b c}+P_{c d}\right)-R_{b a}^{t} P_{b a}\right] \\
0_{(1 \times 3)} & 1
\end{array}\right] \\
T_{E F}=\left[\begin{array}{cc}
R_{c d} & r \cdot\left[R_{b a}^{t}\left(P_{b c}+P_{c d}\right)-R_{b a}^{t} P_{b a}\right] \\
0_{(1 \times 3)} & 1
\end{array}\right]
\end{gathered}
$$

where $T_{B A}$ is the HTM of the right laparoscopic tool, $T_{A B}$ is the inverse of the HTM of the right laparoscopic tool, $T_{C D}$ is the HTM of the left laparoscopic tool, $T_{B C}$ is HTM between the right laparoscopic tool fixed coordinates at point $B$ and the left laparoscopic tool fixed coordinates at C. $T_{A D}$ is the homogenous transformation matrix between the movable coordinates of the right laparoscopic tool at point $A$ and the movable coordinates the left laparoscopic tool at $D$.

$T_{E F}$ is the HTM of the laparoscope, $T_{E B}$ is the HTM between the laparoscope fixed coordinates at $E$ and the right laparoscopic tool fixed coordinates at $B$. It's assumed that the coordinates in the same direction so the rotation matrix is unity matrix and there are only translation in $x, y, z$ directions. $T_{A F}$ is the HTM between the movable right laparoscopic tool coordinates at $A$ and the coordinates at the target point $(F)$ but the translation vector is a ratio $(r)$ of the translation vector of $T_{A D}$. The orientation and position (DKPM) of the laparoscope Camera are calculated directly from $T_{E F}$. The laparoscope parameters are determined in terms of those of the laparoscopic tools [6].

\section{Simulation}

During surgery operation, the laparoscopic tool is moved from one point to another on a certain trajectory. Free and guided trajectories may be selected to avoid obstacles inside the human body. The laparoscopic tools' models are executed using any desired time function for the path of its TCP to perform a certain task. The obtained mathematical models are simulated to show the best positioning of the laparoscope in a surgery environment. The spline trajectory is used to simulate these models [6]. The simulation results show that the laparoscope movements are smooth so the monitor's vision will be stable as shown in Figure 4.

\section{Conclusion}

In this paper, D-H parameters and HTM technique are used to obtain the expressions of the DKPM and IKPM models of two laparoscopic tools and one laparoscope used for its autonomous positioning in laparoscopic surgery. The laparoscopic tools and laparoscope models are used to simulate and align the laparoscope camera with the surgeon's laparoscopic tools' TCPs. The obtained results show a smooth positioning of the laparoscope

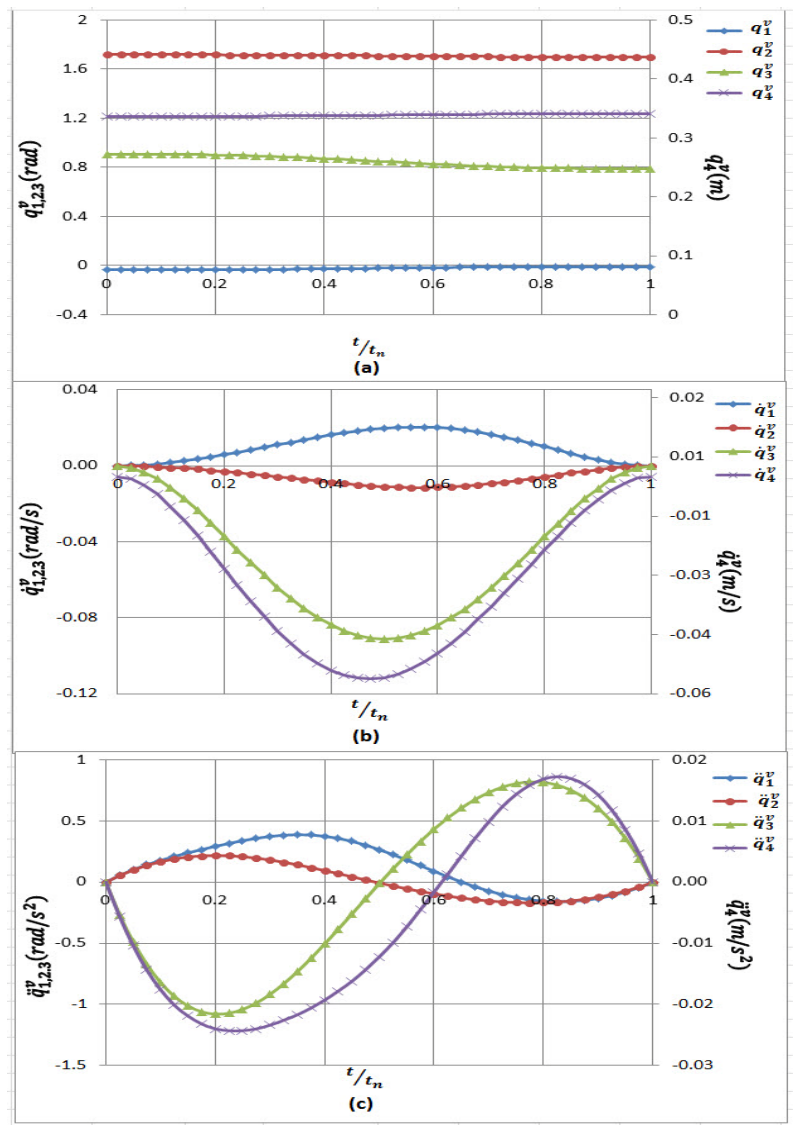

Figure 4. (a) Relation between laparoscope parameters and time ratio; (b) Relation between its derivative and time ratio; (c) Relation between its second derivative and time ratio.

camera for better visualization in laparoscopic surgery environments.

\section{REFERENCES}

[1] N. Halín, P. Loula and P. Aarnio, "Experiences of Using the EndoAssist-Robot in Surgery," Studies in Health Technology and Informatics, Vol. 125, 2007, pp. 161-163.

[2] B. S. Terry, A. D. Ruppert, K. R. Steinhaus, J. A Schoen and M. E. Rentschler, "An Integrated Port Camera and Display System for Laparoscopy,” IEEE Transactions on Bio-Medical Engineering, Vol. 57, No. 5, 2010, pp. 11911197. http://dx.doi.org/10.1109/TBME.2009.2037140

[3] O. Weede, H. Monnich, B. Muller and H. Worn, “An Intelligent and Autonomous Endoscopic Guidance System for Minimally Invasive Surgery,” 2011 ICRA, 2011, pp. 5762-5768.

[4] B. S. Terry, Z. C. Mills, J. a Schoen and M. E. Rentschler, "Single-Port-Access Surgery with a Novel Magnet Camera System," IEEE Transactions on Bio-Medical Engineering, Vol. 59, No. 4, 2012, pp. 1187-1193. http://dx.doi.org/10.1109/TBME.2012.2187292

[5] D. Xu, C. A. A. Calderon, J. Q. Gan, H. Hu and M. Tan, "An Analysis of the Inverse Kinematics for a 5-DOF Ma- 
nipulator," International Journal of Automation and Computing, Vol. 2, 2005, pp. 114-124.

http://dx.doi.org/10.1007/s11633-005-0114-1

[6] S. M. Megahed, "Principles of Robot Modelling and Si- mulation,” John Wiley \& Sons Ltd., 1993, pp. 256-261.

[7] A. Balbola, "Modeling and Simulation of Endoscopes for Autonomously Positioning Videoscope in Laparoscopic Surgery,” Cairo University, 2013. 